Journal of Mathematics and Statistics 5 (4): 360-364, 2009

ISSN 1549-3644

(C) 2009 Science Publications

\title{
On Calculating the Hougaard Measure of Skewness in a Nonlinear Regression Model with Two Parameters
}

\author{
S.A. EL-Shehawy \\ Department of Mathematics, College of Science, Qassim University, P.O. Box 6644, \\ Buriedah 51452, Kingdom of Saudi Arabia
}

\begin{abstract}
Problem statement: This study presented an alternative computational algorithm for determining the values of the Hougaard measure of skewness as a nonlinearity measure in a Nonlinear Regression model (NLR-model) with two parameters. Approach: These values indicated a degree of a nonlinear behavior in the estimator of the parameter in a NLR-model. Results: We applied the suggested algorithm on an example of a NLR-model in which there is a conditionally linear parameter. The algorithm is mainly based on many earlier studies in measures of nonlinearity. The algorithm was suited for implementation using computer algebra systems such as MAPLE, MATLAB and MATHEMATICA. Conclusion/Recommendations: The results with the corresponding output the same considering example will be compared with the results in some earlier studies.
\end{abstract}

Key words: Bias, computer algebra systems, curvature, measures of nonlinearity, nonlinear regression models, skewness

\section{INTRODUCTION}

We consider the usual NLR-model:

$y_{i}=f\left(x_{i}, \Theta\right)+\varepsilon_{i}, i=1,2, \ldots, m$

where, $\left(\mathrm{x}_{\mathrm{i}}, \Theta\right)$ depends on a vector $\mathrm{x}_{\mathrm{i}}$ of regression variables and a vector $\Theta=\left(\theta_{1}, \theta_{2}, \ldots, \theta_{P}\right)$ of unknown parameters to be estimated. This response function $\mathrm{f}$ is a known, scalar-valued function that is twice continuously differentiable in $\Theta$. Here, $y_{i}$ denote to the $i$ th response. The errors $\varepsilon_{\mathrm{i}}, \mathrm{i}=1,2, \ldots, \mathrm{m}$ are usually assumed to be independent and identically distributed normal random variables with mean zero and constant variance $\sigma^{2}$. One can write this assumption as $\varepsilon_{\mathrm{i}} \sim \operatorname{NIID}\left(0, \sigma^{2}\right)$ and refers to this stochastic assumption as an additive error assumption ${ }^{[13,14]}$.

NLR-models differ greatly among themselves with respect to the extent to which the behavior of their least-squares estimators approximates the asymptotic properties. For example, there are some nonlinear regression models whose estimators, even when the sample sizes are relatively small, come close to being unbiased, normally distributed, minimum variance estimators. Such NLR-models are termed close-tolinear models ${ }^{[12-14]}$. Models not possessing these properties may be termed far-from-linear. There are NLR-models that may not be close-to-linear in certain commonly used parameterizations but that, as a result of reparameterizations, become close-to-linear. There are also some NLR-models that never behave in a close-to-linear fashion, even for relatively large sample sizes.

A possible way to analyze the nonlinear behavior of a model data set combination is the calculation of the so called measures of nonlinearity. Previously, a number of measures and procedures of studying the estimation behavior of NLR-models is described. These included the curvature measures of intrinsic and parameter effects nonlinearity, the bias measure of Box and the asymmetry measure of bias of Lowry ${ }^{[1-14]}$. Moreover, a Hougaard measure, which is best to use a direct measure of skewness, was derived in ${ }^{[9]}$.

Computer programs for calculating these measures of nonlinearity are presented in several literatures ${ }^{[5,12,13]}$. Specially, a listing of a FORTRAN-subroutine for computing the Hougaard skewness measure for each parameter in a model appeared in ${ }^{[13]}$.

In this study the Hougaard measure of skewness, which is familiar in statistics, will be considered. We describe this measure and show how this measure is used to indicate a degree of nonlinear behavior for each parameter in a NLR-model. Using MAPLE, a new alternative procedure for calculating the Hougaard measure of skewness of a special two parameter NLRmodel will be presented. 
Description of the Hougarrd measure of Skewness in a NLR-model: Now, we present a description of the studied measure of skewness and explain how to use this measure to indicate a degree of the nonlinear behavior in NLR-models ${ }^{[9,12,13]}$.

Consider a NLR-model (1) with P parameters. Here, the following notations are used to simplify the description method of the Hougaard measure of skewness.

For a nonlinear model:

$\mathbf{f}(\mathbf{x}, \Theta)=\left(f\left(x_{1}, \Theta\right), f\left(x_{2}, \Theta\right), \ldots, f\left(x_{m}, \Theta\right)\right)$

the $\mathrm{m} \times \mathrm{P}$ Jacobian matrix $\mathrm{J}(\hat{\Theta})$ of first derivatives with respect to the parameters is written as:

$\mathbf{J}(\hat{\Theta})=\left[\frac{\mathbf{d} \mathbf{f}(\mathbf{x}, \Theta)}{\mathbf{d} \Theta}\right]_{\Theta=\hat{\Theta}}$

with typical element:

$\mathrm{J}_{\mathrm{ir}}=\frac{\partial \mathrm{f}\left(\mathrm{x}_{\mathrm{i}}, \Theta\right)}{\partial \theta_{\mathrm{r}}}$

evaluated at $\hat{\Theta}$. Also, the $\mathrm{m} \times \mathrm{P} \times \mathrm{P}$ second derivative array (Hessian array) of $\mathbf{f}(\mathbf{x}, \Theta)$ with respect to the parameters is written as $\mathbf{H}(\hat{\Theta})$ with typical elements:

$\mathrm{H}_{\mathrm{irq}}=\frac{\partial^{2} \mathrm{f}\left(\mathrm{x}_{\mathrm{i}}, \Theta\right)}{\partial \theta_{\mathrm{r}} \partial \theta_{\mathrm{q}}}$

evaluated at $\hat{\Theta}$. Here, i runs from 1 to $m$ while $r$ and $q$ run from 1 to $P$.

In matrix notation, each row of $\mathbf{J}(\hat{\Theta})$ is the gradient of one coordinate of $\mathbf{f}(\mathbf{x}, \Theta)$ with respect to $\Theta$ and each face $H_{i}$ of $\mathbf{H}(\hat{\Theta})$ is a complete $P \times P$ second derivative matrix (or Hessian matrix) of one element of $\mathbf{f}(\mathbf{x}, \Theta)$ with respect to $\Theta{ }^{[1,2,9,13,14]}$.

Now, let a term like $L_{r k}, r, k=1,2, \ldots, P$ denote an element of:

$\mathbf{L}=\left[\mathbf{J}^{\mathrm{T}}(\hat{\Theta}) \mathbf{J}(\hat{\Theta})\right]^{-1}$

where, $\mathbf{J}^{\mathrm{T}}(\hat{\Theta})$ is the transpose of the Jacobian matrix $\mathbf{J}(\hat{\Theta})^{[12]}$.

If $\mathrm{W}_{\mathrm{jk} 1}$ is a term defined by:

$$
\mathrm{W}_{\mathrm{jkl}}=\sum_{\mathrm{n}=1}^{\mathrm{m}} \mathrm{J}_{\mathrm{nj}} \mathrm{H}_{\mathrm{nkl}}
$$

then an estimate of the third moment of $\hat{\theta}_{\mathrm{r}}$ is given by:

$$
\begin{aligned}
& \mathbf{E}\left[\hat{\theta}_{\mathrm{r}}-\mathbf{E}\left(\hat{\boldsymbol{\theta}}_{\mathrm{r}}\right)\right]^{3} \\
& =-\left(\mathrm{s}^{2}\right)^{2} \sum_{\mathrm{j}} \sum_{\mathrm{k}} \sum_{1} \mathrm{~L}_{\mathrm{rj}} \mathrm{L}_{\mathrm{rk}} \mathrm{L}_{\mathrm{rl}}\left(\mathrm{W}_{\mathrm{jkl}}+\mathrm{W}_{\mathrm{kjl}}+\mathrm{W}_{\mathrm{lkj}}\right)
\end{aligned}
$$

with the indices $\mathrm{j}, \mathrm{k}$ and $\mathrm{l}$ each ranging from 1 to $\mathrm{P}$. Here $s^{2}$ is the estimate of the residual variance based on the residual sum of squares $\operatorname{RSS}(\hat{\Theta})$ at $\hat{\Theta}$ and also based on (m-P) degree of freedom. It is given by the form $S^{2}=\operatorname{RSS}(\hat{\Theta}) /(m-P) \quad$ with:

$\operatorname{RSS}(\hat{\Theta})=\sum_{i=1}^{m}\left[y_{i}-f\left(x_{i}, \hat{\Theta}\right)\right]^{2}$

The third moment may conveniently be standardized using the appropriate element of the asymptotic covariance matrix $" \mathrm{~s}^{2} \mathbf{L}$ " to give:

$\operatorname{SKEWNESS}_{\theta_{\mathrm{r}}}=\mathbf{E}\left[\hat{\theta}_{\mathrm{r}}-\mathbf{E}\left(\hat{\theta}_{\mathrm{r}}\right)\right]^{3} /\left(\mathrm{s}^{2} \mathrm{~L}_{\mathrm{rr}}\right)^{3 / 2}$

which provides a direct measure of the skewness of $\hat{\theta}_{\mathrm{r}}$.

Because of the close link between the extent of nonlinear behavior of an estimator and the extent of nonnormality in the sampling distribution of the estimator, it is relatively easy to devise a rule-of-thumb for asserting whether the estimator $\hat{\theta}_{\mathrm{r}}$, as assessed by the Hougaard measure SKEWNESS $\theta_{\theta_{\mathrm{r}}}$ for the parameter $\theta_{\mathrm{r}}$, is close-to-linear or contains considerable nonlinearity. Thus, referring to ${ }^{[13]}$ the absolute value $\mid$ SKEWNESS $_{\theta_{\mathrm{r}}} \mid$ of SKEWNESS $\theta_{\theta_{\mathrm{r}}}$ in Table 1 indicates a degree of a nonlinear behavior of the estimator $\hat{\theta}_{\mathrm{r}}$ of the parameter $\theta_{\mathrm{r}}$ in a NLR-model (1).

Application 1: As an application for calculating the Hougaard measure of skewness, we choose the so called Michaelis-Menten model. This model is a model with two parameters and may be written as:

$\mathbf{f}(\mathrm{x}, \Theta)=\frac{\theta_{1} \mathrm{x}}{\theta_{2}+\mathrm{x}}$

where, $\mathbf{f}$ is predicated, $\Theta=\left(\theta_{1}, \theta_{2}\right)$ is a $2 \times 1$ vector of parameters (i.e., $\mathrm{P}=2$ ). This model is an example of a model in which there is conditionally linear parameter $\theta_{1}$. For more details on this chosen model ${ }^{[1,2,5,12,13]}$. 
Table 1: The standard absolute values of Hougaard measure SKEWNESS $_{\theta_{\mathrm{r}}}$ for the parameter $\theta_{\mathrm{r}}$

\begin{tabular}{ll}
\hline SKEWNESS $_{\theta_{\mathrm{r}}} \mid \in(0.00,0.10):$ & $\begin{array}{l}\text { The estimator is very close-to- } \\
\text { linear in behavior. }\end{array}$ \\
$\mid$ SKEWNESS $_{\theta_{\mathrm{r}}} \mid \in(0.10,0.25):$ & $\begin{array}{l}\text { The estimator is reasonably } \\
\text { close-to-linear in behavior. }\end{array}$ \\
$\mid$ SKEWNESS $_{\theta_{\mathrm{r}}} \mid \in(0: 25,1.00):$ & $\begin{array}{l}\text { The skewness is very apparent. } \\
\text { These values indicate }\end{array}$ \\
$\mid$ SKEWNESS $_{\theta_{\mathrm{r}}} \mid \in(1.00, \infty):$ & $\begin{array}{l}\text { considerable nonlinear behavior. } \\
\end{array}$
\end{tabular}

Table 2: The used data set (observations)

\begin{tabular}{lllllll}
\hline $\mathrm{x}$ & 2.0000 & 2.0000 & 0.6670 & 0.6670 & 0.4000 & 0.4000 \\
$\mathrm{y}$ & 0.0615 & 0.0527 & 0.0334 & 0.0258 & 0.0138 & 0.0258 \\
$\mathrm{x}$ & 0.2860 & 0.2860 & 0.2220 & 0.2220 & 0.2000 & 0.2000 \\
$\mathrm{y}$ & 0.0129 & 0.0183 & 0.0083 & 0.0169 & 0.0129 & 0.0087 \\
\hline
\end{tabular}

Table 3: The computed Hougaard measure of skewness for the parameter in the considering model (11) and the given data set

\begin{tabular}{lcl}
\hline Parameter & $\theta_{1}$ & $\theta_{2}$ \\
SKEWNESS $_{\theta_{\mathrm{r}}}$ & 0.989 & 1.180 \\
\hline
\end{tabular}

The working data set in this application consisting of twelve observations (i.e., $m=12$ ) that appeared in ${ }^{[1]}$ are shown in Table 2 . These data yielded least square estimates $\hat{\Theta}=\left(\hat{\theta}_{1}, \hat{\theta}_{2}\right)=(0.1056427059222,1.702689979$ 095) with the residual sum of squares $\operatorname{RSS}(\hat{\Theta})=$ $2.010567 \times 10^{-4}$ at $\hat{\Theta}$ and the estimate of the residual variance $\mathrm{s}^{2}=2.010567 \times 10^{-4} /(12-2)=0.00002010567$ based on 10 degree of freedom ${ }^{[1]}$. By using a listing of a FORTRAN-subroutine for computing the Hougaard measure SKEWNESS $_{\theta_{\mathrm{r}}}$ for the parameter $\theta_{\mathrm{r}}, \mathrm{r}=1,2$ in the considering Michaelis-Menten model and for these given data $^{[1,13]}$, yielding the following values of SKEWNESS $_{\theta_{\mathrm{r}}}$ in Table 3.

With regard to Ratkowsky's results in Table 3, it is clear that the skewness for the estimator of the parameter $\theta_{1}$ is very apparent and the value of the skewness for the estimator of the parameter $\theta_{2}$ indicates considerable nonlinear behavior. This means that the estimators of the two parameters in this example are far-from-linear in their estimation behavior.

Now, we will compute again the values of the Hougaard measure of skewness SKEWNESS $_{\theta_{\mathrm{r}}}$ for the parameter $\theta_{\mathrm{r}}, \mathrm{r}=1,2$ in the model (11) but by using an alternative suggested computational algorithm with MAPLE-procedure and the results will be compared with the previous obtained values of the Hougaard measure of skewenees in Table 3.
A suggested algorithm to compute the Hougaard measure of Skewness in a NLR-model with two parameters: To calculate the Hougaard measure of skewness for the illustrative example with respect to the NLR-model with two parameters (11) in Application 1, an alternative algorithm is explained and its MAPLEprocedure is formulated using MAPLE 9.5 as follows:

\section{Algorithm 1:}

Step 1: Describe the parameter space as two dimensional spaces.

Step 2: Define the considering 2-parameter model as model equation.

Step 3: Define the expectation surface as a $(1,12)$ vector through calculation of the model function for each mass-point.

Step 4: Define the Jacobian-matrix "JAC" (i.e., J( $\hat{\Theta})$ ) of the model function with respect to the parametervector and calculate this matrix for the estimated parameters.

Step 5: Define "L" as the inverse matrix of the multiplication $\left[\mathbf{J}^{\mathrm{T}}(\hat{\Theta}) \mathbf{J}(\hat{\Theta})\right]$ of the transpose matrix $\mathbf{J}^{\mathrm{T}}(\hat{\Theta})$ and the matrix $\mathbf{J}(\hat{\Theta})$.

Step 6: Define a (12, 2, 2)-tensor (Hessian matrix) "HES" (i.e., H( $\hat{\Theta})$ ) of the 2 nd order derivatives of the model function with respect to the parameter-vector and calculate this matrix for the same estimated parameters.

Step 7: Formulate the terms "W [j, k, l]"(i.e., $\left.\mathrm{W}_{\mathrm{jkl}}\right)$ as a multiplication form in (7) of the Jacobian matrix JAC and the Hessian matrix HES.

Step 8: Formulate the third moment "TM[i]" (i.e., $\left.\mathbf{E}\left[\hat{\theta}_{i}-\mathbf{E}\left(\hat{\theta}_{i}\right)\right]^{3}\right)$ of the parameter $\theta_{i}$ as in (8).

Step 9: Formulate the Hougaard measure of skewness "SK[i]" (i.e., SKEWNESS $\theta_{\theta_{i}}$ ) of the parameter $\theta_{i}$ as it appeared in (10).

Step 10: Give the using variables $x$, $\hat{\Theta}$ (i.e., "Par") and $\mathrm{s}^{2}$ (i.e., "ssq") in the procedure to proceed the calculation for each element in the previous steps with respect to the considering model.

Step 11: Evaluate the Hougaard measure SKEWNESS $_{\theta_{i}}$ for each ith parameter. 
The results of the previous algorithm provide two values. The first value is SKEWNESS $_{\theta_{1}}$ and the second one is SKEWNESS $_{\theta_{2}}$.

The proposed MAPLE-procedure to calculate the Hougaard measure of skewness for the parameter $\theta_{\mathrm{r}}$, $\mathrm{r}=1,2$ in the model (11) with its output will be given below. To verify the aim of the calculation for the Hougaard measure of skewness through the previous algorithm, we consider the data in Table 1 with the obtained least square estimates $\hat{\Theta}$ and also the obtained estimate of the residual variance $\mathrm{s}^{2}$ as above. With this suggested MAPLE-procedure, the program produces the values 0.9887703882 and 1.180088401 of SKEWNESS $_{\theta_{1}}$ and SKEWNESS $_{\theta_{2}}$ respectively. In addition, these results are identical to the previous results in Table 3 which were obtained from a listing of a FORTRAN-subrotine for computing the Hougaard skewness measure in ${ }^{[13]}$.

\section{MATERIALS AND METHODS}

The so called Michaelis-Menten model with two parameters have been selected to apply our alternative computational algorithm through its proposed procedure for calculating the values of the Hougaard measure of skewness. The procedure is formulated by using MAPLE 9.5 as follows:

\section{A MAPLE-procedure for calculating the Hougaard measure of skewness:}

$>$ restart: Digits: $=10$ : with(linalg):

$>\mathrm{SK}:=\operatorname{proc}(\mathrm{X}:$ :vector, par::vector, ssq::float)

local i, j, k, p, s, c, l, r, x, J, H, JAC, HES, HESS, W, TM, L;

global Theta , m, z, f, F, SK;

$\mathrm{m}:=\operatorname{vectdim}(\mathrm{X}): \mathrm{z}:=2:$ Theta: $=\operatorname{array}(1 \ldots \mathrm{z}): \mathrm{f}:=(\mathrm{x}$, Theta)->Theta[1]*x/(Theta[2]+x);

$\mathrm{F}:=\operatorname{array}(1 \ldots \mathrm{m}): \mathrm{J}:=\operatorname{array}(1 \ldots \mathrm{m}): \mathrm{H}:=\operatorname{array}(1 \ldots \mathrm{m})$ :

for i from 1 to $m$ do

$\mathrm{F}[\mathrm{i}]:=\mathrm{f}(\mathrm{X}[\mathrm{i}]$, Theta);

od:

for $\mathrm{c}$ from 1 to $\mathrm{m}$ do

$\mathrm{J}[\mathrm{c}]:=$ jacobian (vector([F[c]]), [Theta[1], Theta[2]]);

$\mathrm{H}[\mathrm{c}]:=\operatorname{hessian}(\mathrm{F}[\mathrm{c}]$, [Theta[1], Theta[2]]);

od:

JAC: $=\operatorname{subs}($ Theta[1] $=\operatorname{par}[1]$, Theta[2]=par[2], stackmatrix (J[1], J[2], J[3], J[4], J[5], J[6],

$\mathrm{J}[7], \mathrm{J}[8], \mathrm{J}[9], \mathrm{J}[10], \mathrm{J}[11], \mathrm{J}[12]))$;

$\mathrm{L}:=$ inverse (evalm (transpose (JAC)* JAC)):

HES: $=\operatorname{subs}(\operatorname{Theta}[1]=\operatorname{par}[1]$, Theta[2] $=\operatorname{par}[2]$,
Stackmatrix (H[1], H[2], H[3], H[4], H[5], H[6], H[7], $\mathrm{H}[8], \mathrm{H}[9], \mathrm{H}[10], \mathrm{H}[11], \mathrm{H}[12])$ );

for $\mathrm{s}$ from $\mathrm{z}$ by $\mathrm{z}$ to $\mathrm{z}^{*} \mathrm{~m}$ do

HESS[s/2]: =submatrix (HES, s-1 .. s, 1 .. z);

od:

for 1 from 1 to $\mathrm{z}$ do

$\mathrm{W}[1,1,1]:=\operatorname{sum}(\mathrm{JAC}[\mathrm{r}, 1] * \operatorname{HESS}[\mathrm{r}][1,1], \mathrm{r}=1 . . \mathrm{m})$;

$\mathrm{W}[1,2,1]:=\operatorname{sum}(\mathrm{JAC}[\mathrm{r}, 1] * \operatorname{HESS}[\mathrm{r}][2,1], \mathrm{r}=1 . . \mathrm{m})$;

$\mathrm{W}[2,1,1]:=\operatorname{sum}(\mathrm{JAC}[\mathrm{r}, 2] * \operatorname{HESS}[\mathrm{r}][1,1], \mathrm{r}=1 . . \mathrm{m})$;

$\mathrm{W}[2,2,1]:=\operatorname{sum}(\mathrm{JAC}[\mathrm{r}, 2] * \operatorname{HESS}[\mathrm{r}][2,1], \mathrm{r}=1 . . \mathrm{m})$;

od:

$\mathrm{i}:=\mathrm{i}^{\prime}: \mathrm{j}:=^{\prime} \mathrm{j}$ ':

for i from 1 to $\mathrm{z}$ do

TM[i]: = -((ssq)2)*sum(sum(sum(L[i, j]*L[i, k ]*L[i, $\left.\mathrm{p}]^{*}(\mathrm{~W}[\mathrm{j}, \mathrm{k}, \mathrm{p}]+\mathrm{W}[\mathrm{k}, \mathrm{j}, \mathrm{p}]+\mathrm{W}[\mathrm{p}, \mathrm{k}, \mathrm{j}]), \mathrm{j}=1 . . \mathrm{z}\right)$, $\mathrm{k}=1 . . \mathrm{z}), \mathrm{p}=1 . . \mathrm{z})$;

od :

i:=' i ':

for i from 1 to $\mathrm{z}$ do

SK[i]:=TM[i] $/\left(\left(\operatorname{ssq}^{*} L[\mathrm{i}, \mathrm{i}]\right)^{\wedge}(3 / 2)\right)$ :

od;

$\operatorname{print}($ SKEWNESS[theta[1]]=SK[1]);

print(SKEWNESS[theta[2]]=SK[2]);

end:

$>\mathrm{X}:=\operatorname{vector}([\mathrm{2}, 2,0.667,0.667,0.4,0.4,0.286$, $0.286,0.222,0.222,0.2,0.2])$ :

> par:=vector([ 0.1056427059222, 1.702689979095 ]):

ssq:= $0.00002010567: \mathrm{SK}(X$, par, ssq);

$$
\begin{gathered}
\text { SKEWNESS }_{\theta_{1}}=0.9887703882 \\
\text { SKEWNESS }_{\theta_{2}}=1.180088401
\end{gathered}
$$

Also, we used the data set which consisting of twelve observations and the results have been compared with the results which were obtained from a listing of a FORTRAN-subrotine. The obtained least square estimates $\hat{\Theta}$ of $\Theta=\left(\theta_{1}, \theta_{2}\right)$ by using these data with the value of $\operatorname{RSS}(\hat{\Theta})$ at $\hat{\Theta}$ and the estimate of the residual variance $\mathrm{s}^{2}$ based on 10 degree of freedom was considered.

\section{RESULTS}

Our alternative suggested computational algorithm with MAPLE-procedure gave values of the Hougaard measure of skewenees SKEWNESS $_{\theta_{1}}=0.9887703882$ and SKEWNESS $_{\theta_{2}}=1.180088401$ which are identical to the previous obtained values of this studied measure by using a listing of a FORTRAN-subrotine. 


\section{DISCUSSION}

Our study presented an alternative computational algorithm for calculating the Hougaard measure of skewness as a nonlinearity measure in a NLR-model with two parameters. These obtained values indicate a degree of a nonlinear behavior in the estimator of the parameter in the studied model. Our suggested algorithm was applied on an example of a NLR-model in which there is a conditionally linear parameter. This proposed algorithm was applied using MAPLE.

By using optimal accuracy in the application the proposed algorithm with its procedure will be very effective. The suggested algorithm is available for other NLR-models which have not any conditionally linear parameter and in this case the used procedure must be changed in some functions. Similarly one can also used the same algorithm with a suitable procedure for NLRmodels with numbers of parameters more than two.

\section{CONCLUSION}

The described algorithm in this study with its MAPLE-procedure is very effective by using optimal accuracy. The suggested algorithm with its considering MAPLE-procedure indicates a method for calculating the Hougaard measure of skewness as a nonlinearity measure in a two parameter NLR-model, in which there is conditionally linear parameter. This proposed method is competitive to other methods with the same aim which appeared in previous literature.

\section{REFERENCES}

1. Bates, D.M. and D.G. Watts, 1980. Relative curvature measures of nonlinearity (with discussion). J. R. Stat. Soc. Ser., 42: 1-25. http://www.jstor.org/stable/2984733

2. Bates, D.M. and D.G. Watts, 1988. Nonlinear Regression Analysis and its Applications. John Wiley and Sons, Inc., New York, ISBN: 0471816434, pp: 365.

3. Bates, D.M., D.C. Hamilton and D.G. Watts, 1983. Calculation of intrinsic and parameter effects curvatures for nonlinear regression models. Commun. Stat. Simulat. Comput., 12: 469- 477. http://www.informaworld.com/smpp/ftinterface?co ntent $=\mathrm{a} 780059743 \& \mathrm{rt}=0$ \& format $=\mathrm{pdf}$

4. Box, M.J., 1971. Bias in nonlinear estimation. J. R. Stat. Soc. Ser., 33: 171-201. http://www.jstor.org/ pss/2985002
5. El-Shehawy, S.A. and A.A. Karawia, 2007. An alternative computational algorithm for calculating the nonlinearity of regression models with two parameters. Applied Math. Comput., 188: 686-692. http://cat.inist.fr/?aModele $=$ afficheN\&cpsidt $=1876$ 8597

6. Guay, M., 1995. Curvature measures for multiresponse regression models. Biometrika, 82: 411-417. http://www.jstor.org/stable/2337418

7. Haines, L.M., T.E.O. 'Brien and G.P.Y. Clarke, 2004. Kurtosis and curvature measures for nonlinear regression models. Stat. Sincia, 14: 547570.

http://direct.bl.uk/bld/PlaceOrder.do?UIN=149150 $338 \&$ ETOC $=$ RN\& from $=$ searchengine

8. Hamilton, D.C., D.G. Watts and D.M. Bates, 1982. Accounting for intrinsic nonlinearity in nonlinear regression parameter inference regions. Ann. Stat., 10: 386-393. http://www.jstor.org/pss/2240673

9. Hougaard, P., 1985. The appropriateness of the asymptotic distribution in a nonlinear regression model in relation to curvature. J. R. Stat. Soc. Ser., 47: 103-114. http://www.jstor.org/pss/2345550

10. Lowry, R.K. and R. Morton, 1983. An asymmetry measure for estimators in non-linear regression models. Proceeding of the 44th Session International Statistics Institution, Madrid, Contribution, (SIMC'83), Artes Graf. Danubio, Madrid, pp: 351-354.

11. Morton, R., 1987. Asymmetry of estimators in nonlinear regreesion. Biometrika, 74: 679-685. http://www.jstor.org/stable/2336461

12. Ratkowsky, D.A., 1983. Nonliear Regression Modeling. Markcel Dekker, New York, ISBN: 0824719077, pp: 276.

13. Ratkowsky, D.A., 1990. Handbook of Nonlinear Regression Models. Markcel Dekker, New York, ISBN: 0824781899, pp: 241.

14. Seber, G.A.F. and C.J. Wild, 2005. Nonlinear Regression. John Wiley and Sons, New York, ISBN: 10: 0471617601, pp: 800. 